\title{
COMPLEX OF HELIOGEOPHYSICAL INSTRUMENTS OF NEW GENERATION
}

\author{
G.A. Zherebtsov \\ Institute of Solar-Terrestrial Physics SB RAS \\ Irkutsk, Russia,gaz@isz.irk.ru
}

\begin{abstract}
The paper discusses the importance of the problem of adverse effects of cosmic processes and phenomena (space weather factors) on the ground engineering and technical infrastructure, space-born radioelectronic facilities, etc.

It examines the state of the experimental base for research in solar, atmosphere, and near-Earth space physics in Russia and abroad. The necessity of creating newgeneration instruments, developed using advanced engineering solutions and technologies, is justified.

The complex of the main facilities and instruments
\end{abstract}

of the National Heliogeophysical Complex of the Russian Academy of Sciences is analyzed. The main scientific directions of basic research and applied problems are formulated.

Keywords: space weather, solar-terrestrial relations, monitoring, forecast, geophysical effects, solar activity.

\section{INTRODUCTION}

By the late 1980s, the Institute of Solar-Terrestrial Physics of Siberian Branch of the Russian Academy of Sciences (ISTP SB RAS) had the most advanced material and technical experimental facilities in Russia, which enabled us to make continuous regular monitoring and measurements, to conduct research in solar-terrestrial physics, to address crucial applied problems. The groundbased heliogeophysical infrastructure included eight observatories located in the Irkutsk Region, the Krasnoyarsk Territory, and the Republic of Buryatia. For solar physics research, ISTP SB RAS developed the Automated Horizontal Solar Telescope, the Large Solar Non-Eclipse Coronagraph, the Infrared Telescope AZT-33IK, the Solar Telescope for Operational Predictions, the Large Solar Vacuum Telescope, several chromospheric telescopes, the Siberian Solar Radio Telescope. For cosmic ray research, a cosmic ray spectrograph and a network of cosmic ray stations were deployed. The geomagnetic field and physical processes in the magnetosphere were observed and studied using a meridional chain of magnetic stations; comprehensive studies of high-latitude geophysical processes in the ionosphere and magnetosphere were conducted at Norilsk Observatory, equipped with ionosondes, riometers, magnetometric equipment, optical devices, and other instruments. For research in ionospheric and radio wave propagation physics, along with the use of vertical sounding stations and optical instruments the Institute deployed a network of experimental radio paths in Siberia and Far East, developed special facilities for research into dynamic processes in the ionosphere.

On the basis of the radar station, passed on to the Institute under the Soviet Military Conversion Program, an incoherent scatter radar was developed and modernized. It is Russia's unique instrument so far, which is used not only for conducting ionospheric and magnetospheric research, but also for solving problems of space debris.
The basic elements of the main facilities were, however, developed in the 1960-80s, and many of them cannot be changed fundamentally; hence the need for radical modernization of all the experimental facilities. The logic of science development and the need for practice pose new scientific problems whose solution requires innovative approaches to studies of the Sun, nearEarth space, Earth's upper atmosphere, and their related processes. To achieve scientific progress it is necessary to consider the Sun-Earth system as unified with complex internal relations and interactions.

Addressing this important problem calls for not only satellite studies, but also for the development of new ground-based infrastructure. There is a need for new large experimental facilities, which have not been developed in our country over the past 35-40 years, complexes of instruments with advanced capabilities for measurements and experiments. To attain the level of the highest world standards in this area of research and to ensure the development strategy for the next 25-30 years, it is necessary to develop such facilities and complexes in the years to come.

In order to promote basic research in solar-terrestrial physics and near-Earth space focused on solving topical applied problems of space weather in the interests of the economy and national security protection, to develop new, competitive space technologies including dual-use ones, ISTP SB RAS on behalf of the President of the Russian Federation and the corresponding Decree of the Government of the Russian Federation has prepared a consolidated investment project National Heliogeophysical Complex of the Russian Academy of Sciences (NHC RAS), which has been approved by the President of the Russian Academy of Sciences. The project involves developing the scientific and research structure of the new generation complex as part of ISTP SB RAS, which will enable us to take a quantum leap in experimental research in solar and near-Earth space physics, to meet the challenges of developing and assimilating new space technologies, including double-purpose ones. 


\section{RELEVANCE OF FUDAMENTAL HELIOGEOPHYSICAL RESEARCH AND APPLIED PROBLEMS}

Near-Earth space (NES), which comprises such regions as Earth's upper atmosphere (UA), ionosphere, and magnetosphere, is a space whose physical conditions depend on solar and geomagnetic activity.

In recent decades, this space has not only been studied purposefully and massively — it has become an area of intense practical activity. In NES there are a large number of spacecraft (SC) of different purposes used for research in the interest of the national economy, for solving applied problems, specifically in the interest of the national security. Reliability and efficiency of SC operation are defined both by the use of the latest technologies and by the state of the medium surrounding the facilities.

It is therefore essential to have complete information on the processes occurring in NES, the possibility of diagnosing and predicting cosmic phenomena (space weather), as well as assessing the possible consequences.

In NES, strong disturbances occur from time to time which are driven by intense fluxes of charged particles or by magnetized plasma streams ejected from the solar surface (solar wind). These disturbances cause spacecraft failures. They may appear as surface electrification of spacecraft or extensive electrification due to penetration of energetic particle fluxes into SC. Under conditions of disturbed near-Earth plasma, the impacts on SC can be enhanced when SC passes over areas of inhomogeneous magnetospheric-ionospheric plasma. Analysis of SC failures clearly shows that one of the main causes of electronic equipment failures are extreme conditions of space weather. The most adverse factors are driven by powerful solar disturbances - firstly, by coronal mass ejections, solar flares, and other eruptive processes. Also of great importance is the solar magnetic field carried from its surface to the interplanetary space. Solar eruptive phenomena produce shock waves in the solar wind plasma, which evolve as they propagate from the Sun to Earth's orbit, forming shock flows representing an interplanetary shock wave having a complex plasma-magnetic structure. Interacting with the geomagnetic field, the solar wind leads to the formation of a magnetic cavity - magnetosphere - around Earth. Under quiet conditions, boundaries of the magnetosphere shield the geomagnetic field from solar wind plasma. Under certain conditions, a small amount of solar wind energy, however, penetrates into the magnetosphere and in the form of corpuscular streams is injected into the high-latitude atmosphere, generating a magnetospheric substorm, which is characterized by the occurrence of auroras at high latitudes and short-term restructuring of the geomagnetic field in the outer magnetosphere. Shock solar wind streams affecting the magnetosphere trigger longer intense atmospheric disturbances - geomagnetic storms generating noticeable fluctuations of the geomagnetic field even at middle and lower latitudes.

The above geophysical phenomena significantly affect technological objects in space and on Earth. Adverse effects of space weather disrupt the normal opera- tion of such facilities, telecommunication networks, navigation systems, electric transmission lines, and pipelines. The rapid development of technosphere and its growing expansion to space lead to the fact that space weather poses the highest risks to the economy infrastructure. This problem is particularly important and calls for special attention in the Arctic, which is a kind of kitchen for space weather on the planet. Here, both the NES responses to extreme solar phenomena and the effects of the interaction of global and wave phenomena in the atmosphere with regional dynamic structures are most pronounced.

Of crucial importance at high latitudes are electrodynamic processes in ionospheric plasma during its interaction with the magnetosphere. They are most conspicuous in geomagnetic storms when intense energetic particle precipitation occurs, powerful electric fields and currents are generated. The result is a strong Joule heating of UA, restructuring of its dynamic mode, significant changes of parameters, development of ionospheric plasma instabilities generating irregularities of different scales, etc. Highlatitude ionospheric disturbances propagate to middle latitudes. In this case, due to mismatch between geographic and magnetic poles the spatial and temporal patterns of evolution of the disturbances are very complex and are still the subject of research.

Especially relevant are the studies of the structure and interaction of the mid-latitude and subarctic stratosphere, the mesosphere, and the lower thermosphere as an important link in the vertical transfer of energy and momentum in the atmosphere. Mesospheric phenomena are the most sensitive indicator of climatic temperature changes. It becomes necessary to know features of this region to develop aerospace and radio communication technologies. UA density perturbations in the lower mesosphere can affect the trajectory of rocket launches, change the map of probable drop areas of spent rocket parts. Tidal and internal atmospheric waves propagating through the lower and upper atmosphere cause ionospheric parameters to change, thereby affecting the radio communication system. To improve the accuracy and reliability of wireless communication systems, radar, and satellite navigation, it is important to monitor wave activity in the mesosphere and lower thermosphere.

The continuous development of technologies employed in the Arctic zone, the use of high-sensitive communication means require a continuous improvement of environmental monitoring and prediction systems. The extended territory of the Russian Federation (the longitudinal sector of which exceeds $100^{\circ}$ ) requires us to monitor the magnetosphere-ionosphere-atmosphere interaction in order to obtain a global picture of disturbances during various extreme events on the Sun, in Earth's magnetosphere and atmosphere. The NES state in the Arctic exerts a decisive effect on the performance and efficiency of vitally important technological systems of energy, navigation, radio communication with regional centers, ships, planes, including safety and security of high-latitude routes. In connection with the intensification of works on the economic development in the Arctic, and specifically extraction of natural resources, the tasks of developing high-performance moni- 
toring, warning, and communication systems, as well as provision of security of the country move to the forefront in the national and economic activity.

The space environment is not only explored, but also its artificial modification is studied by various methods with a particular purpose - to deteriorate or improve its state depending on a problem to solve. In foreign countries, the issues concerning artificial modification, control over processes in NES, use of artificial geophysical effects for developing new double-purpose technical facilities have been actively studied. It is, therefore, essential to have complete information about the processes occurring in NES, and to distinguish natural processes from effects of artificial influence on NES.

It is necessary to have an opportunity to estimate these natural phenomena (space weather) and to take measures to reduce their possible consequences. Ensuring high reliability and efficiency of space and groundbased equipment requires a deep understanding of the physical processes occurring on the Sun and in NES.

Solar activity and its prediction on all time scales have become important factors in maintaining the technological infrastructure in NES and on Earth. Note that currently available models of solar activity influence on NES are not yet accurate enough for real-time assessment of risks and efficiency of equipment in outer space and on Earth.

The system of geophysical support of diagnostics and prediction of disturbances in NES is based on diagnosing and predicting the main geoeffective phenomena of solar activity. Coronal plasma ejections usually reach Earth in 2-3 days; and the most powerful ones, in $15 \mathrm{hrs}$. Arriving at Earth, they trigger geomagnetic storms, which affect communication, induce currents and electric potentials in distributed energy networks, etc. Solar flares generate bursts of electromagnetic radiation, powerful flares, disrupt the operation of spacecraft, ground-based radars, navigation and communications systems in a wide frequency range. The bursts reach Earth in $8 \mathrm{~min}$, mainly affecting its daylight side. Fluxes of secondary ionized particles within tens of minutes after flares lead to an increase in radiation in NES, which is dangerous to people in spacecraft and aircraft during transpolar flights and causes malfunction and destruction of spacecraft electronics.

The diagnostics of disturbances involves monitoring the electromagnetic solar radiation in optical and radio bands; they can be predicted only if we have a sufficiently complete understanding of physical processes, develop physically based models and predictive algorithms. The main reason for the lack of adequate models of evolution of the active region in which non-stationary processes and geoeffective phenomena occur is the lack of knowledge about physics of solar processes on a spatial scale $\sim 50-100 \mathrm{~km}$. Solar plasma parameters on this scale can be measured only using telescopes with an aperture $2-4 \mathrm{~m}$, hence the need for large solar optical telescopes.

The diagnostic potential of solar radio observations has not fully exploited so far because of the absence of radioheliographs with high temporal, spatial, and spectral resolution.
The largest new-generation telescopes have been deployed and developed abroad over recent years. The USA, China, Brazil have developed projects of the most advanced multiwave radioheliographs, which are currently at different stages of implementation. The European consortium has started developing a four-meter optical solar telescope; construction of such a telescope in the USA is virtually completed. In Germany, a telescope GREGOR with a $1.5 \mathrm{~m}$ mirror has been developed and deployed on the Canary Islands. France and Italy have designed a telescope virtually free of instrumental polarization to study magnetism and variability of the Sun. Since 2003, in the USA a special-purpose telescope for synoptic studies of the Sun has been working; it is intended to run for 25 years. Moreover, a great number of new-generation large experimental facilities and observatories for studying geophysical processes have been developed. For example, in the USA there are heating facilities in Alaska (HAARP) and in Puerto Rico (Arecibo Heating Facility) for ionosphere modification by powerful HF radio waves. The USA has worked out and replicated a mobile incoherent scatter radar (IS radar) AMISR; on Svalbard, an IS radar ESR. Near the magnetic pole, a polar cusp observatory is being built (the USA, Canada). Around the North and South Poles, the international network of large coherent HF radars SuperDARN is deployed. It is designed to study the magnetosphere-ionosphere coupling. Thirteen of the radars belong to the USA. Several mesospheric-stratospherictropospheric (MST) radars have been built. Projects of a super high-power equatorial MST IS radar (Japan) and a multi-position system of new-generation IS radars of the European Incoherent Scatter Scientific Association EISCAT have been developed. To date, the leading geophysical centers have been formed as clusters of instruments, which combine a set of radio and optical facilities around IS radars. These facilities allow for the most comprehensive diagnostics of charged and neutral components of the upper atmosphere. Their capabilities greatly complement and extend networks of magnetic stations. Such clusters have a status of international or national centers.

At present, the Russian Federation is in a critical position in the field of geophysical research. The situation can be attributed to several factors, but the main of them consists in undervaluation of the importance of in-depth studies aimed at solving problems of application of NES, its influence on the development of new space technologies, including dual-use ones. The backlog is most pronounced in the development of ground-based research infrastructure, as compared to international standards. This is due to the fact that until recently the Russian Federation did not develop large facilities (telescopes, radioheliographs, IS and MST radars, lidars, etc.).

It should be noted that in the USA such research clusters are developed and used under the National Space Weather Program, which provides the country's readiness to short-term and long-term effects of cosmic natural phenomena, which cannot be avoided but should be studied in depth, predicted, and reduced. 
A national space weather program having similar aims and objectives of obtaining real-time and reliable information on the state of the heliogeophysical environment should also be developed in our country. Its ultimate strategic goal is to create an effective scientificbased national system of warnings and voice alerts about dangerous cosmic phenomena for respective services to take necessary preventive measures and to reduce adverse effects.

This national program should consist of two conceptually related parts:

- organization of basic research with the use of space- and ground-based instruments targeted at specific applications;

- organization of a ground-based network of necessary heliogeophysical observations and measurements involving special space-based facilities.

\section{OBJECTIVES}

\section{AND INSTRUMENTAL COMPOSITION OF THE NATIONAL HELIOGEOPHYSICAL COMPLEX OF THE RUSSIAN ACADEMY OF SCIENCES}

The Heliogeophysical Complex of the Russian Academy of Sciences includes five unique large experimental instruments for research in the field of solar and NES physics, which are to be developed and manufactured mainly in industrial enterprises of the Russian Federation:

- Large Solar Telescope-Coronagraph with a mirror $3 \mathrm{~m}$ in diameter (LST-3);

- Multiwave Radioheliograph;

- Radiophysical Complex for Atmospheric and Ionospheric Research (RPhC);

- Network of Coherent Ionospheric Radars (SECIRA);

- Lidar Optical Complex (LOC).

\subsection{The Large Solar Telescope-Coronograph with a mirror $3 \mathrm{~m}$ in diameter (LST-3)}

The modern Large-aperture Solar optical Telescope (LST-3) [Grigoryev et al., 2020] is a complex engineering facility. The development of large-aperture telescopes is driven by the need to study physical processes on the Sun with extremely high spatial resolution corresponding to only a few kilometers on the solar surface because on these very scales the main magnetohydrodynamic processes determining solar luminosity variations in different spectral ranges occur.

Solar phenomena cover large areas of the solar surface and are associated with an imbalance in the largescale solar magnetic field. The nature of energy accumulation and the triggering mechanism of the imbalance lie in the fine structure of solar magnetism. It is precisely the microstructure of solar magnetic fields that plays an essential role in physics of large-scale phenomena of solar activity, which have important geoeffective effects.

Essential elements of these new telescopes without which even the large size of mirrors cannot provide ad- equate spatial resolution are adaptive optics (AO) systems, experience of development of which for solar observations is now completely absent in Russia. Such systems require specific matrix detectors, very complex deformable mirrors, high-speed special-purpose computers, complex mathematical algorithms. There is therefore no doubt that the acquisition of experience in development and operation of such AO systems will fill the technology gap in Russia in this field, which is also of great practical importance.

LST-3 equipped with an AO system will allow observations of different atmospheric layers and the solar corona with spatial resolution, polarization and photometric accuracy unattainable before. This will enable us to address the problems associated with energy release in plasma dynamic phenomena on the Sun and corona heating processes. This, in turn, will allow us to assess the state of the interplanetary medium, the level of radiation exposure of SC and high-altitude aircraft, especially along subauroral paths.

The program of works on LST-3 involves developing quantitative models of basic solar activity phenomena and determining optimal quantitative and qualitative parameters of solar active processes as input parameters for algorithms of short- and medium-term forecast of solar activity. The large size of the telescope aperture will allow us to actively use it for nighttime observations. Particularly effective are observations of lowluminous objects, especially in geostationary orbits. Using AO at night will significantly increase resolution of the instrument and make it possible to determine the spatial structure of individual space objects. It is important to coordinate LST-3 with other observation instruments of the Heliogeophysical Complex under construction and with the currently available telescopes (AZT-33IK, AZT-33VM) of the ISTP SB RAS Sayan Astrocomplex.

The operation of the telescope in the coronographic mode will allow us to observe space objects such as asteroids and comets near the Sun including hazardous ones in the daytime. This will greatly increase capabilities for monitoring NES.

Main areas of research carried out with LST-3:

- elaboration of the model of the large-scale magnetic field in the corona, calculation of characteristics of the interplanetary magnetic field and heliospheric current sheet; prediction of magnetic field parameters in Earth's orbit;

- designing a model of evolution of solar active regions and methods of medium-term prediction during active region development;

- development of a model of pre-flare state of an active region, elaboration of the solar flare model, methods for predicting flares and their energy parameters;

- development of a dynamic model of coronal mass ejections and kinematics of their movement in the interplanetary space, algorithms for predicting coronal mass ejection effects in near-Earth space;

- improvement of models for solar flux variability in different spectral ranges; 
- nighttime observations of low-luminous space objects and spacecraft.

Thus, the development of LST-3 should make a decisive contribution to our understanding of the origin of solar activity, which drives space weather phenomena. This will contribute to the solution of the most important applied problems.

\subsection{The multiwave radioheliograph and the complex of spectropolarimeters}

Important, and in many ways unique, information on the processes occurring in the solar atmosphere is provided by radio observations with special-purpose radio telescopes whose distinctive features are all-weather capability and relatively low cost of instruments.

The most dynamic processes determining NES disturbances are dynamic processes of magnetic fields in the solar corona. Currently, the only method of measuring coronal magnetic fields is the analysis of radio emission spectra and polarization. This requires measurements at high temporal, spatial, and spectral resolution.

The development of radioheliographs [Altyntsev et al., 2020] with the required characteristics has been made possible due to the appearance of analog broadband fiber lines, improvement of the element base of UHF electronics, reduction of prices of computing with simultaneous increase in its performance, attainment of high accuracy in positioning of antenna elements with the aid of space technologies.

The radioheliograph will allow us to develop the existing methods of identifying the source and starting point of coronal mass ejections on the solar disk from shading of local areas of the disk. Note that the duration of the shading, until the plasma emission is still dense enough, is of the order of ten minutes, so to detect such effects in sequences of radio maps and to use the data in the dynamic model of coronal mass ejections and kinematics of their movement in the interplanetary space require significant computational resources.

Observations at high spectral resolution will allow for continuous magnetography of coronal magnetic fields and pre-identification of magnetic structures, explosive dissipation of magnetic fields in which causes solar flares. Through interactive fitting of the spatial structure and spectral characteristics of model radio sources, calculated from photospheric magnetograms, and images in an optical range to the spectral and spatial characteristics actually observed with the radioheliograph, we can significantly increase the accuracy of determining characteristics of coronal magnetic fields. Consequently, the reliability and accuracy of solar flare prediction based on models of pre-flare state of active regions will increase considerably. In the case of shortterm prediction, the required frequency of provision of such combined maps is several maps per day.

Along with solar disk observations, the radioheliograph with high spatial resolution will monitor the integral solar emission in a unique wide frequency range 0.05-150 GHz, thus enabling us to calculate our own (not Canadian) index $F 10.7$ describing radio emission of the quiet Sun at a frequency of $2.8 \mathrm{GHz}$. The wide range of received frequencies will allow us to elaborate the model of solar emission variability in different spectral ranges and to develop additional radio indices of solar activity, useful in solving specific prediction problems.

Emission monitoring, analysis of its spectral composition and power of radio bursts, during which the electromagnetic radiation power may exceed the background level millions of times, will enable us in some cases to find the causes of failures in SC electronic systems, radars, navigation and communication systems. Generalization and systematization of such diagnostic information are very useful for hardware developers. The results of solar activity monitoring will be available online.

The classification of flares by identifying prognostic signs of powerful solar flares (pre-bursts), generation of shock waves (type II bursts), and accelerated electron fluxes (type III bursts), proton fluxes (millimeter emission during flares) will allow it to be used online by the national forecast center.

Mapping of microwave sources with the Multiwave Radioheliograph in a frequency band 3-24 GHz will enable us to localize various structures in the solar atmosphere, including active regions, filaments, and coronal holes. The resulting information on the position of the solar disk radio brightness center can be used for navigation. Mapping of active regions in circular polarization will be used in the developed methods of short-term prediction of powerful solar flares and in the model of active region evolution. Of particular interest is the prediction of flare activity for appearing and disappearing active regions near the solar limb, not observed by optical telescopes. Managing the list of received frequencies allows us to select monitoring modes, i.e. to observe either fast processes with a limited frequency range at one-second resolution or quasi-stationary coronal structures at high spectral resolution. For example, for magnetography of active regions image series with a sufficiently small step in receiving frequency, i.e. with high spectral resolution, can be obtained for several minutes. The duration of observations depends on the length of daylight up to $6 \mathrm{hrs}$ in winter and $10 \mathrm{hrs}$ in summer.

Thus, the Multiwave Radioheliograph implements all-weather monitoring of solar activity processes in the solar atmosphere, results of which are necessary for prediction and diagnostics of geoeffective solar activity effects.

All-weather monitoring of explosive processes on the Sun will enable us to solve an important applied problem - to assess their impact on the operation of space facilities, as well as ground-based communication, navigation, radar, and other technology systems. A mathematical basis for solving this problem is the development of quantitative models of the main solar activity phenomena, the determination of the optimal quantitative and qualitative parameters of active solar processes as input parameters for short-term and mediumterm forecast of solar activity.

The spectropolarimeters developed under the project 
and the geographically dispersed network of stations deployed on their basis will significantly improve the quality of prediction of geoeffective solar activity effects. Particularly promising is the deployment of two complexes of spectropolarimeters in subpolar regions. This will allow for round-the-clock monitoring of geoeffective solar activity effects and for refinement of the predictive model of magnetic field parameters in Earth's orbit.

As a result of the monitoring, in the radio frequency band the models of the large-scale magnetic field in the solar corona will be refined, the interplanetary magnetic field and the heliospheric current sheet will be calculated.

Thus, we can formulate the fundamental research directions and applied problems that will be implemented with the radioheliograph:

- topology of coronal magnetic fields during the active and quiet Sun;

- identification of regions responsible for magnetic field conversion;

- wave processes and shock waves in the solar atmosphere;

- evolution of large-scale structures in the solar atmosphere during the 11-year solar cycle;

- regular measurements of radio emission at a wavelength of $10.7 \mathrm{~cm}$;

- radio emission monitoring in a wide frequency range to apply this data to, for example, communication, radar, radio navigation systems, etc.;

- assessment of geoeffectiveness of solar flare parameters;

- short-term prediction of powerful solar flares with a lead time of 2 to 3 days.

\subsection{The radiophysical complex for ionospheric} and atmospheric research

In the project considered, the Radiophysical Complex $(\mathrm{RPhC})$ is the largest complex and multifunctional informational system designed to solve problems of ionospheric and atmospheric physics, controlled modification of the ionosphere by powerful radio waves, and study of the effect of NES physical processes on technology systems.

Structurally, RPhC consists of the main cluster of instruments, which includes the most powerful and promising research instruments: a radio wave incoherent scatter (IS) radar for ionospheric sounding, a mesospheric-stratospheric-tropospheric (MST) radar for sounding the neutral atmosphere [Medvedev et al., 2020], and a heating facility for modifying the ionosphere by powerful HF radio waves [Vasilyev et al., 2020a]. RPhC also comprises a network of coherent ionospheric radars (SECIRA) [Berngardt et al., 2020].

This cluster of large research measurement facilities will be supplemented with a system of small problemoriented instruments and a meridional chain of stations Norilsk-Irkutsk (ionosondes, magnetometers, photometers, etc.).

$\mathrm{RPhC}$ is a multipurpose complex allowing fast transition to new challenges. Research directions would change due to the development of research into the upper atmosphere (UA) and solar-terrestrial relations. There are no facilities of this type in Russia nowadays, therefore a wide range of scientific questions related to the atmosphere dynamics is not supported by up-to-date high-precision experimental data. The modern IS-MST radar in Irkutsk will compensate for the lack of experimental data in this field of research.

The location of $\mathrm{RPhC}$ is unique since the complex will provide important geophysical data and monitor NES in the center of Russia, significantly complementing observational data acquired by geophysical centers in the USA, Europe, and Japan in studying global distributions of environmental parameters.

The main focus of RPhC studies is on the upper atmosphere $(80-1500 \mathrm{~km})$ as one of the most important parts of the Sun-Earth system, which plays a key role in the interaction between ionized and neutral gaseous mantles of Earth. On the one hand, these processes are determined by solar emission and plasma mechanisms of energy conversion and momentum transfer in the solar wind - magnetosphere-ionosphere - thermosphere

chain. On the other hand, the UA energy, structure, and dynamics also depend on planetary and tidal oscillations, internal atmospheric waves, and turbulent processes generated in the lower layers of the atmosphere, i.e. near Earth's surface and ocean area.

$\mathrm{RPhC}$ is fundamentally important in studying this interaction by performing complex complementary measurements of parameters of ionized and neutral UA components with $\mathrm{RPhC}$ and the Lidar Optical Complex (LOC). The MST radar will play a crucial role in these studies, measuring parameters of the atmosphere in an altitude range $10-90 \mathrm{~km}$. This efficient method will be first implemented in our country with $\mathrm{RPhC}$. This will allow using $\mathrm{RPhC}$ and LOC to study all atmospheric layers as a unified system, which is one of the main directions of the development of atmospheric research.

A priority and rapidly developing direction will be laboratory experimental research on modification of the ionosphere by powerful HF radio waves. It will allow for a more detailed study of ionospheric and UA parameters, issues of controlled influence on processes in the ionosphere and NES. In RPhC for such studies, a heating facility will be developed which is comparable with the leading foreign analogues in diagnostic facilities and energy potential.

$\mathrm{RPhC}$ will provide a unique opportunity to study radio wave propagation, including nonlinear, with simultaneous highly informative diagnostics of ionospheric plasma.

The results of the ionospheric and UA research with $\mathrm{RPhC}$, the possibility of using it for highly informative monitoring of NES are important for various fields of science and technology: space and terrestrial radio communication, radar, and navigation; SC operation; NES monitoring, including problems of comet and asteroid impact hazards and space debris.

2.3.1. The Incoherent Scatter Radar and the Magnetospheric-Stratospheric-Thermospheric Radar (ISMST radar)

The IS-MST radar [Medvedev et al., 2020] under conditions of natural noise background, actual electromagnetic and geophysical conditions in the deployment area will ensure remote sounding of the ionosphere, mesosphere, stratosphere, and troposphere at an altitude 
range of 10 to $2000 \mathrm{~km}$ over the point of the radar location at $\pm 45^{\circ}$ beam deviation from the normal in any angular coordinate.

The multipurpose IS-MST radar will address a set of basic problems of atmospheric research.

Interaction between the magnetosphere and the ionosphere-thermosphere system

The UA interaction with the magnetosphere is determined by a complex of plasma processes that have a strong effect on the UA structure and dynamics especially during geomagnetic storms when at auroral latitudes energetic particle precipitation occurs, intense magnetospheric electric fields and currents are generated. This induces the thermospheric Joule heating and pressure gradients, which cause strong neutral winds and internal gravity waves (IGW) propagating to the equator and altering the thermospheric circulation system. As a result, the neutral composition changes, the plasma moves along magnetic field lines, thus altering UA ionization and recombination conditions. At the same time, the neutral winds due to the dynamo effect generate electric fields, which, in turn, affect plasma and neutral gas. Thus, the ionized and neutral components interact nonlinearly, largely determining the UA dynamics and electrodynamics.

A particular problem is the UA response to superstorms when the mid-latitude ionosphere in their basic parameters and structure becomes close to the auroral one. The mechanisms of penetration of electric fields and precipitating energetic particles into so low latitudes, occurrence of ionospheric plasma instabilities should be studied.

The IS-MST radar will enable us to address these issues by studying in detail the structure and dynamics of the ionized and neutral components of mid-latitude UA during storms, propagation of disturbances from high to middle and low latitudes.

\section{Effect of lower layers of the neutral atmosphere on UA}

The UA dynamics is largely determined by planetary waves and IGW of its neutral component, which have a wide range of spatial (from a few to thousands of kilometers) and temporal (from several minutes to several days) scales. Sources of these waves are diverse - meteorological processes in the troposphere, Joule heating by ionospheric currents, explosions, earthquakes, etc. The ionospheric response to IGW manifests itself as traveling ionospheric disturbances (TIDs) whose radiophysical research has been carried out for many years. Many pressing questions, however, remain unanswered: the determination of IGW spectrum portions that make the major contribution to the UA thermal regime, energy, and dynamics; the wave transfer effect on the chemical composition of the mesosphere and thermosphere; the IGW effect on generation of electric fields and plasma instabilities; the IGW interaction with tides and neutral winds and their effect on variations in UA parameters; the identification of IGW sources, etc.

These problems can be solved only with the IS-MST radar capable of measuring parameters of IGW of neutrals and TIDs, determining their spatial-temporal dis- tributions at spaced points, monitoring TID propagation with the help of the meridional chain of auxiliary instruments, as well as using observational data from regional and global networks of stations.

\section{Global changes and regional characteristics}

Global changes and long-period trends of variations in environmental parameters are among the current problems. In the atmospheric research, data series on changes in all atmospheric layers, which can be obtained with the IS-MST radar, are most important.

An advanced research direction is a comprehensive study of the lithosphere-ionosphere-atmosphere relations. The Irkutsk region is characterized by high seismic activity, which necessitates research into atmospheric and ionospheric manifestations of this activity based on data from the IS-MST radar and seismic stations.

The East Siberian region has important global features: the greatest difference between magnetic and geographic latitudes and a strong magnetic anomaly, which once again underlines the uniqueness of the location of the IS-MST radar. It is in this region where in recent years many extremely powerful UA disturbances have been recorded, in particular during moderate geomagnetic storms, which requires finding causes for this phenomenon.

Radio wave propagation and radiophysical methods for remote ionosphere diagnostics

Ionospheric plasma as a radio wave propagation medium features irregularities of different scales, dispersion, absorption, anisotropy, and, under certain conditions, nonlinearity. Inhomogeneous electrical properties of the earth surface, its difficult topography and cover also exert a profound effect on radio wave properties. For different wavelengths and different radio paths, these properties of the medium show up as diverse effects, which in many cases occur in totality and affect the radio wave propagation process in a complicated way. Study of radio wave propagation in such complex media is one of the most important problems of radiophysics.

Various effects of the interaction between radio waves and the ionosphere allow us to find the connection between its parameters and radio signal characteristics. On this basis, methods of remote ionosphere diagnostics have been worked out. Radiophysical methods provide the largest amount of data on the UA structure and properties. Development of these methods and enhancement of their diagnostic capabilities are therefore among the important directions.

The IS-MST radar will be used for research into ionospheric, including nonlinear, propagation of $\mathrm{HF}$, VHF, and EHF radio waves. The advantage of the ISMST radar is its capability of performing highly informative diagnostics of ionospheric plasma.

This will allow us to develop new methods of remote diagnostics of the environment, which are based on the analysis of specific signal realizations, rather than of their statistical characteristics. The main attention here will be paid to coherent and interference diagnostic methods. 
Thus, capabilities of the multipurpose IS-MST radar will allow for new fundamental research and, in addition, for solution of a number of important applied problems such as

- effective monitoring of current geophysical conditions to ensure the operation of radio aids in Southeastern Siberia, to lay the necessary groundwork for the development of effective models for predicting adverse geophysical events;

- development of new control technologies for low-orbit spacecraft and space debris with simultaneous diagnostics of ionospheric conditions;

- development of new radio super-resolution methods based on the principles of precision signal processing and interferometry with a small base, which will enable us to achieve high accuracy in the determination of parameters of LEO space objects (SO). The new methods will allow us to detect and track $10 \mathrm{~cm}$ SO over distances up to $1000 \mathrm{~km}$ with range accuracy $\sigma_{R} \sim 100 \mathrm{~m}$, angular accuracy $\sigma_{\varepsilon}$ and $\sigma_{\gamma} \sim 1$ arcmin;

- designing integrated highly informative radaroptical space monitoring methods capable of detecting and determining SO paths with the IS-MST radar with subsequent setting of telescopes with a narrow field of view on a selected SO.

The simultaneous and coordinated monitoring of SO will allow for its portraiture, significantly increase the accuracy and reliability of non-coordinate information on SO, including events of destruction, separation of small fragments, onboard engine operation, and disorientation.

\subsubsection{The heating facility}

Studies on the interaction of high-power HF radio waves with ionospheric plasma are extensive and intensively developing branch of UA physics. The controlled influence on the ionosphere has opened an entirely new opportunity for controlled active experiments enabling us to study properties of the ionosphere and UA more deeply than passive measurements, to conduct studies on the controlled influence on ionospheric and NES processes, which are aimed at examining and controlling processes in UA. Heating or modification of the ionosphere by powerful $\mathrm{HF}$ radio waves leads to heating of electrons and occurrence of various plasma instabilities, which give rise to various phenomena and effects.

The heating generates large-scale temperature and electron density perturbations, defocusing (E layer) and focusing (F layer) lenses, cross-modulation, and selfaction of radio waves. In the powerful standing radio wave field, periodic artificial irregularities (grids) are formed radio detection of which allows us to measure a number of parameters of plasma and neutral components.

Amplitude modulation of a powerful wave causes modulation of conductivity of the ionosphere and currents, induced by external electric fields (at high latitudes) or by the neutral wind (at middle latitudes). This current is a source of artificial VLF-ELF emission, which propagates in the Earth-ionosphere waveguide over large distances (up to thousands of kilometers) or in the whistler mode in the magnetosphere. This effect can be used for subsurface radar and for modifying the magnetosphere.

The instabilities excite plasma waves, layers, filaments, artificial ionospheric turbulence, i.e. ionospheric plasma irregularities of various types. One of the most significant effects is a pronounced small-scale plasma layering into the irregularities strongly elongated along the magnetic field. Artificial airglow in the E and F layers and artificial broadband ionospheric radio emission, as well as excitation (enhancement) of plasma and ionic lines in signal spectra measured by IS radars are of particular importance.

This area of research is one of the most promising. It is essential for the study of UA natural properties, for example, conditions for the occurrence of instabilities [Vasilyev et al., 2020a].

In addition, the heater can be used for

- experiments on the evaluation of efficiency of $\mathrm{HF}$ radio wave energy transfer to ionospheric plasma; study of plasma formations resulting from such impact, their location, lifetime, secondary radio and optical radiation, shape, degree of irregularity, etc.;

- study of effects of HF, VHF, and UHF radio waves through a disturbed region, capabilities of generating ELF radio waves through conductivity modulation in ionospheric plasma;

- study of phenomena associated with generation of magnetospheric VLF waves and controlled stimulation of magnetic substorms and particle precipitation from Earth's radiation belts.

\subsection{Network of coherent ionospheric radars (SECIRA)}

Study of the processes occurring under the influence of the solar wind on Earth's magnetosphere and ionosphere is one of the central problems of solar-terrestrial physics. One of the most effective instruments for addressing this problem is the international collaborative system SuperDARN (Super Dual Auroral Radar Network). It is a network of high-frequency (shortwavelength) radar of coherent backscattering whose field of view covers polar regions in Northern and Southern hemispheres. On the basis of Doppler measurements, this network can determine the picture of convection of polar ionosphere plasma being an important indicator of its interaction with the magnetosphere. Currently there are 23 radars in the Northern Hemisphere and 13 in the Southern Hemisphere, which encompass polar regions.

ISTP SB RAS has been deploying the Russian network of coherent ionospheric radars analogous to $\mathrm{Su}$ perDARN. Russian radars can make observations almost over the entire territory of the Russian Federation and are capable of studying the magnetosphereionosphere coupling, including effects of magnetospheric substorms and geomagnetic storms in mid-latitude and subauroral regions.

The Russian system of coherent radars involves studies in several major areas in the framework of international cooperation, and under independent programs [Berngardt et al., 2020]. 
Solar wind interaction with the magnetosphere and ionosphere

The main problem of the interaction between the solar wind and the magnetosphere is to estimate the electric field of reconnection and its variations along the socalled confluence line, i.e. along the projection of the reconnection region at the magnetopause on the ionosphere. The currently available systems can do this under certain conditions, but are limited to a fairly small observation interval. There is a need to cover most of the daytime sector. A contribution these radars will make in Russia will be the expansion of the radar field of view to all local time intervals, thereby removing the above limitation.

Large- and medium-scale convection of auroral plasma is one of the important parameters for studying the magnetosphere-ionosphere coupling. The Russian radars can significantly reduce the existing gap in the longitudinal range of observations and can study the magnetosphere-ionosphere coupling, including effects of magnetospheric substorms and geomagnetic storms in mid-latitude and subauroral regions, intensively explored today.

\section{Internal atmospheric waves}

While at middle latitudes the main source of internal atmospheric waves (IAW) are influences on the ionosphere from below and transition processes in the solar terminator, at high latitudes an important source of large-scale internal waves in the upper atmosphere is a strong Joule heating in the high-latitude ionosphere, which is related to either substorms and reinforced electrojets or to enhanced ionospheric flows in cusp. Such waves propagating in the upper atmosphere generate TIDs, which focus and defocus propagating highfrequency radio waves. SuperDARN radars regularly observe large-scale and medium-scale TIDs. The Russian radars will increase the field of view of the system and facilitate the search and experimental study of large-scale waves, which are important for quantitative estimation of the energy transfer in the lithosphereatmosphere - ionosphere - magnetosphere system.

\section{Scattering by meteors and mesospheric winds}

These radars will allow us to observe meteor scattering of radio waves, thus measuring winds in the mesosphere. Expansion of the network at the expense of the Russian segment will allow for measurements in all longitudinal sectors simultaneously. Such measurements provide for monitoring and study of medium-scale structures in mesospheric winds with high spatial and temporal resolution and wide spatial coverage.

\section{Natural and artificial plasma irregularities}

Coherent radars receive coherent backscattering from ionospheric irregularities in the $\mathrm{E}$ and $\mathrm{F}$ layers of the ionosphere. These irregularities are generated primarily by electric fields and electron density gradients in these regions. These radars can therefore be used for studying processes responsible for generation of these irregularities. In addition to the irregularities that have a natural origin, of great interest are artificial irregularities stimulated by such powerful sources of radio waves as the heating facility «EISCAT Heating» (Tromsø, Norway). While the Russian HF radars cannot cover the regions in which the existing foreign heating facilities are located, Russian scientists will, however, have an opportunity to use data from other radars in their future studies.

\section{ULF waves}

$\mathrm{HF}$ radars can make measurements of ULF waves in several ways, in particular in the range of periods of Pc5 geomagnetic pulsations (150-600 s). They can either directly measure ionospheric disturbances, caused by the wave electric field, or record disturbances of parameters of backscatter sounding signals, driven by motion of the reflecting ionospheric layer induced by the wave electric field. Location of the Russian radars is unique as it will allow for measurements of waves in the vicinity of the plasmapause, which is one of the most important regions of near-Earth space.

In addition to fundamental research, the Russian network SECIRA will provide the solution to the following applied problems:

- continuous monitoring of ionospheric disturbances in sub-polar regions affecting the operation of communication, navigation, radar, and other technological systems over the entire territory of Russia;

- monitoring of the auroral oval boundary providing an experimental basis for efficient prediction of blackouts in HF radio channels during strong geomagnetic disturbances and prediction of optimal operating frequencies under different geophysical conditions;

- implementation of near real-time diagnostics of wave ionospheric disturbances as most unpredictable factor of disturbances for communication, radar and navigation systems.

As a result, the Russian network of coherent decameter radars, which includes four rapid deployment mobile radars with a field of view covering a significant part of the Russian Arctic will solve the problem of warning of the federal and regional executive authorities, radio communication and navigation systems, ESIMO (Roshydromet) about adverse space weather factors emerging in the Arctic.

\subsection{Lidar optical complex (LOC)}

Nowadays, the development of global and regional systems of atmospheric research at various altitude levels is a challenging fundamental task whose accomplishment involves scientific and technical potentials of the global community.

The Lidar Optical Complex (LOC) [Matvienko et al., 2020] is designed to study profile characteristics of physical parameters (temperature, density, wind) and composition (a number of gas components, aerosol) of the middle and upper atmosphere, which are formed under the influence of natural processes and anthropogenic effect (e.g., environmental disruptions, strong radiation). The optical complex combines active and laser systems and passive optical instruments for recording the intrinsic optical emission of atmospheric components. This complex will provide the most comprehensive information on the state of the atmosphere. 
Lidar measurements with LOC will enable qualitatively new studies of conditions and composition of the atmosphere in a wide range of heights from 10 to $100 \mathrm{~km}$. Different sounding methods in use provide information on heliogeophysical processes and phenomena occurring in this height range. This information gives insight into the influence of solar activity, magnetospheric conditions, stratospheric warming events, gravity and planetary waves, volcanic and seismic activity, polar and noctilucent clouds. As is known, UA comprising charged particles is explored with radiophysical complexes, and the neutral atmosphere may be examined with lidar systems. Combination of these systems will allow for the analysis of the relationship between neutral and charged components, which is one of the key problems in Earth's atmosphere physics. These issues are still poorly understood and are of fundamental and practical importance.

Study of the upper atmosphere, including the mesosphere and the thermosphere, requires lidar systems with large optics and high-power lasers. Such a mesostratospheric lidar will be developed for the first time in our country as part of NHC. It will enable us to determine stratospheric and mesospheric parameters at high spatial and temporal resolution. We should be able to obtain these parameters simultaneously with results of radio-physical measurements, which is essential for the study of solarterrestrial relations.

The Mesostratospheric Lidar with the use of theoretical and experimental research developments, received at V.E. Zuev Institute of Atmospheric Optics of Siberian Branch of the Russian Academy of Sciences (IAO SB RAS) with sounding techniques, will allow us to solve the problems of remote identification of the basic atmospheric parameters: temperature, density, wind speed, ozone content, distribution of sodium vapor in the mesosphere.

Thus, the use of new technologies and developed methods of measurements with the Mesostratospheric Lidar will allow for continuous monitoring of thermodynamic parameters and aerosol-gas components of the mesosphere and stratosphere and will provide information on the atmosphere in an altitude range from 10 to $100 \mathrm{~km}$.

It should be emphasized that this lidar system allows for its further modernization and elaboration.

The lidar is an active instrument comprising powerful transmitters and large optics. In the project presented, it will be supplemented with a set of passive instruments capable of measuring density, temperature, speed, and wave propagation characteristics at heights of the mesosphere and lower thermosphere. The spectral composition and regular variations of the main emission lines and bands in US in long time intervals have been studied well enough. This allowed us to turn to the development of empirical models of regular variations in the main UA emissions.

The passive instruments include the following [Vasilyev et al., 2020b]:

- Fabry - Perot interferometer, which will be used to study the dynamics of the mesosphere, thermosphere, plasma drifts, as well as to measure the relative density, temperature, wind and wave velocity;
- all-sky cameras, which are necessary to obtain intensity distributions of the main optical emissions at mesospheric and thermospheric levels with high spatial resolution;

- photometers, which can measure the main optical emissions of the mesosphere and lower atmosphere with high temporal and amplitude resolution;

- spectrographs, which can measure spectral contents of emission and temperature.

Variations in UA are a sensitive indicator of physical-chemical processes and its main parameters. A connection has been established between airglow and such phenomena as sudden winter stratospheric warming events, earthquakes, etc. Airglow observations are of great scientific interest for studies of the atmosphere and Earth's climate. Another important practical application is the use of the intrinsic optical emission in experiments on ionosphere modification.

Thus, LOC will allow for the solution of urgent basic problems in atmospheric research at different height levels, which today is also the most important practical problem in the field of environmental ecology and global climate change.

Moreover, NHC includes another important object. Collection, real-time processing, and storage of information from instruments of the complex, as well as its transfer to users will be performed at the center which will be established in Irkutsk (ISTP SB RAS). Its computing capabilities and hardware and software architecture should ensure compliance with the requirements for speed, storage capacity (at least 100000 terabytes per year), development of necessary warnings, indices, and other indicators of the state of the Sun and NES.

\section{CONCLUSION}

The National Heliogeophysical Complex under construction, which includes unique facilities, instruments, and devices, has been developed on the basis of new technical solutions with the use of modern technologies and meets international standards.

The experimental base developed will allow for the transition to a qualitatively new level of development of basic and applied research in the field of solar-terrestrial physics (physics of the Sun, Earth's atmosphere, ionosphere, and magnetosphere), and will ensure the implementation of these works at a high level for the next 2530 years.

Deployment of this complex at or near the ISTP SB RAS network of observatories will allow for the coordination and joint experiments with the existing scientific infrastructure.

Potential scientific and technical capabilities of the complex of new generation instruments in solving fundamental and applied problems should become the basis for the development of a scientific and technical program of research into negative space phenomena and reduction of their consequences, along with planned space experiments.

The geographical location of the complex, its multifunctionality and technical equipment will enable Russian scientists to participate in international programs and projects in the field of space research. 
The research complex can be a center of education, training, and research activities for Russian and foreign students, graduate students, researchers, as well as a training center for scientists and engineers for research organizations and universities.

The developed conception of NHC RAS provides for the establishment of a Federal Research Center for fundamental and applied research in solar and NES physics in view of the currently available research infrastructure of ISTP SB RAS with the participation of other associated scientific organizations.

ISTP SB RAS has submitted a draft project of this center to the Ministry of Science and Higher Education of the Russian Federation.

\section{REFERENCES}

Altyntsev A.T., Lesovoi S.V., Globa M.V., Gubin A.V., Kochanov A.A., Grechnev V.V., et al. Multiwave Siberian Radioheliograph. Solar-Terr. Phys. 2020, vol. 6, no. 2, pp. 30-40. DOI: $10.12737 /$ stp-62202003.

Berngardt O.I., Kurkin V.I., Kushnarev D.S., Grkovich K.V., Fedorov R.R., Orlov A.I., et al. ISTP SB RAS decameter radars. Solar-Terr. Phys. 2020, vol. 6, no. 2, pp. 63-73. DOI: $10.12737 /$ stp-62202006.
Grigoryev V.M., Demidov M.L., Kolobov D.Yu., Pulyaev V.A., Skomorovsky V.I., Chuprakov S.A., and the AMOS team. Project of the Large Solar Telescope with mirror $3 \mathrm{~m}$ in diameter. Solar-Terr. Phys. 2020, vol. 6, no. 2, pp. 14-29. DOI: $10.12737 /$ stp-62202002.

Matvienko G.G., Marichev V.N., Bobrovnikov S.M., Yakovlev S.V., Chistilin A.Yu., Sautkin V.A. Mesostratospheric Lidar for the Heliogeophysical Complex. Solar-Terr. Phys. 2020, vol. 6, no. 2, pp. 74-83. DOI: 10.12737/stp-62202007.

Medvedev A.V., Potekhin A.P., Setov A.G., Kushnarev D.S., Potekhin A.P., Lebedev V.P. All-atmosphere IS-MST Radar. SolarTerr. Phys. 2020, vol. 6, no. 2, pp. 41-48. DOI: $10.12737 /$ stp62202004.

Vasilyev R.V., Setov A.G., Frolov V.L., Ratovsky K.G., Beletsky A.B., Oinats A.V., et al. Modern heating facility for research into the mid-latitude ionosphere. Solar-Terr. Phys. 2020a, vol. 6, no. 2, pp. 49-62. DOI: 10.12737/stp-62202005.

Vasilyev R.V., Artamonov M.F., Beletsky A.B., Zorkaltseva O.S., Komarova E.S., et al. Scientific goals of optical instruments of the National Heliogeophysical Complex. Solar-Terr. Phys. 2020b, vol. 6, no. 2, pp. 84-97. DOI: 10.12737/stp-62202008.

How to cite this article

Zherebtsov G.A. Complex of heliogeophysical instruments of new generation. Solar-Terrestrial Physics. 2020. Vol. 6. Iss. 2. P. 3-13. DOI: $10.12737 /$ stp-62202001. 